\title{
Associations of Alcohol
} Dehydrogenase and Aldehyde Dehydrogenase Polymorphism With Cognitive Impairment Among the Oldest-Old in China

\section{OPEN ACCESS}

Edited by:

Wael M. Y. Mohamed,

International Islamic University

Malaysia, Malaysia

Reviewed by:

Zhixiong Sun,

Columbia University, United States

Lilach Soreq,

University College London,

United Kingdom

Jaya Kumar,

National University of Malaysia,

Malaysia

*Correspondence:

Lijing Yan

lijing.yan@dukekunshan.edu.cn

Chenkai Wu

chenkai.wu@dukekunshan.edu.cn

${ }^{\dagger}$ These authors have contributed equally to this work

Specialty section:

This article was submitted to Neurocognitive Aging and Behavior,

a section of the journal

Frontiers in Aging Neuroscience

Received: 17 May 2021

Accepted: 28 December 2021

Published: 25 February 2022

Citation:

Jin $X$, Long $T$, Chen $H$, Zeng $Y$,

Zhang X, Yan L and Wu C (2022)

Associations of Alcohol

Dehydrogenase and Aldehyde

Dehydrogenase Polymorphism With

Cognitive Impairment Among

the Oldest-Old in China.

Front. Aging Neurosci. 13:710966.

doi: 10.3389/fnagi.2021.710966

\begin{abstract}
Xurui Jin ${ }^{1,2 \dagger}$, Tingxi Long ${ }^{1 \dagger}$, Huashuai Chen ${ }^{3}$, Yi Zeng ${ }^{3,4}$, Xian Zhang ${ }^{1,2}$, Lijing Yan ${ }^{1,5,6 *}$ and Chenkai $W^{1 *}$

${ }^{1}$ Global Health Research Center, Duke Kunshan University, Kunshan, China, ${ }^{2}$ MindRank Al Ltd., Hangzhou, China, ${ }^{3}$ Center for the Study of Aging and Human Development and Geriatrics Division, Medical School of Duke University, Durham, NC, United States, ${ }^{4}$ Center for Healthy Aging and Development Studies, National School of Development, Peking University, Beijing, China, ${ }^{5}$ School of Health Sciences, Wuhan University, Wuhan, China, ${ }^{6}$ The George Institute for Global Health, Beijing, China
\end{abstract}

Recent literature suggested that $A L D H 2$ mutation is associated with alcohol metabolism, and ethanol intake might jointly increase the risk of Alzheimer's disease (AD) in mice. However, it is unclear whether this synergistic effect exists among humans. We examined the associations of four single nucleotide polymorphisms (SNPS) on aldehyde dehydrogenase $(A L D H)$ and alcohol dehydrogenase $(A D H)$ genes (i.e., $A L D H 2$ rs671, ADH1B rs1229984, ADH1B rs1042026, and ADH1C rs1693482) and cognitive impairment among the oldest-old. We also investigated whether this association was modified by ethanol intake from alcohol consumption. Data were from the Chinese Longitudinal Healthy Longevity Survey genetic sub-study, including 1,949 participants aged over 90 years. Participants with a Mini-Mental State Examination (MMSE) score of $<18$ were considered cognitively impaired. Alcohol consumption was categorized as heavy, moderate, or never drinkers. With the dominant model, carrying A allele on rs671, C allele on rs1229984, and T allele on rs1042026 was associated with 33\% (95\% confidence interval [Cl]: 5\%, 69\%), 33\% (95\% Cl: 2\%, 75\%), and 29\% (95\% Cl: 3\%, 62\%) higher odds of cognitive impairment in the multivariable-adjusted logistic model, respectively. We did not observe a significant interaction between those SNPs and alcohol consumption. Among the oldest-old, carrying ALDH2 rs671 mutation was associated with higher odds of cognitive impairment independent of alcohol consumption.

Keywords: ethanol intake, Asian flush, alcohol metabolizing genes, Alzheimer's disease, cognitive impairment

\section{INTRODUCTION}

Aging is a major contributor to cognitive decline and dementia across the world, and it is becoming a major public health concern. Previous studies have discussed demographic or socioeconomic risk factors for dementia among old adults, with limited attention on genetic risk factors using large samples. Most of the genetic research on cognitive function focused on candidate genes which have been demonstrated to be associated with Alzheimer's disease (AD) or identified by genome-wide 
association studies (GWAS). Those candidate genes include apolipoprotein E (APOE), catechol-O-methyltransferase (COMT) (Komulainen et al., 2008), brain-derived neurotrophic factor (BDNF) (Bray et al., 2005), and dystrobrevin-binding protein 1 (DTNBP1) (Wray et al., 2008), with the APOE $\varepsilon 4$ allele being by far the strongest genetic risk factor and accounts for about $5 \%$ of the variance in lifetime cognitive change and $4 \%$ of the variance in $\mathrm{AD}$. In addition to those genetic factors, some recent works have also indicated the key role of some alcohol metabolism-related genes as enzymes involved in the detoxification of the ethanol metabolism in the pathology of AD.

Alcohol dehydrogenase $(A D H)$ and aldehyde dehydrogenase $(A L D H)$ are the two major alcohol-metabolizing enzymes (Chen et al., 2014). The mutation of several single nucleotide polymorphisms (SNPs) on those two genes results in the change of enzymatic activity, such as rs671 in $A L D H$, rs1693482 in $A D H 1 C$, and rs1229984 and rs1042026 in ADH1B (Hurley and Edenberg, 2012). Among the Asian population, the prevalence of $A D H 1 B, A D H 1 C$, and $A L D H 2$ mutation is performed. It is estimated that nearly $30 \%$ of people in Asia ( $~ 8 \%$ of the world population) carry the genetic variants of the $A L D H 2$ A allele by rs671 (Eng et al., 2007). Previous literature has indicated that a reduced activity of $A D H$ and $A L D H$ would lead to an excess of acetaldehyde and result in oxidative stress and mitochondrial dysfunctions which have been identified in both familial and sporadic AD (Zhang and Ren, 2011; Chen et al., 2012; Swerdlow, 2018). At the population level, $A D H$ and $A L D H$ genes have been shown to be associated with alcohol dependence (Macgregor et al., 2009) - a leading cause of dementia (Koch et al., 2019). At present, the associations between $A L D H 2$ and $A D H$ genetic polymorphisms with cognitive function or $\mathrm{AD}$ were inconclusive. Some studies suggested that ALDH2 (rs671 polymorphism) is a risk factor for AD in Japanese (Kamino et al., 2000), whereas others reported no association. A recent study including $339 \mathrm{AD}$ patients and 168 healthy controls investigated the association of several SNPs on $A D H$ and $A L D H$ with $\mathrm{AD}$ and found a suggestive association between $A D H 1 C$ rs2241894 and $\mathrm{AD}$ among women (Wu et al., 2021). However, some other genetic studies have found that the negative impact of genetic factors may be cumulative by age, such as $A P O E$ gene on cognitive function, and there is a fewer study of $A D H$ and $A L D H$ genes among the oldestold population.

A recent experimental study suggested that chronic excessive ethanol intake and the $A L D H 2$ gene mutation might jointly increase the risk of $\mathrm{AD}$ in mice (Joshi et al., 2019). Alcohol consumption, as one of the major risk factors of brain damage, is associated with the incidence of dementia. There is a J-shaped association of alcohol consumption with dementia, where excessive alcohol intake or abstinence increased dementia risk, compared with consuming 9-112 g/week (Topiwala and Ebmeier, 2018). In a large cross-sectional study from Southern China, occasional rather than moderate alcohol use was found to be associated with better cognitive function (Au Yeung et al., 2011), suggesting that the observed effects could be driven by a complex interaction between alcohol and other factors. In systematic reviews, drinking patterns are associated with $\mathrm{AD}$ and also cognitive function. It is unclear whether $A L D H 2$ mutation is associated with cognitive function and whether this mutation and ethanol intake synergistically contribute to the development of $\mathrm{AD}$ among humans.

The aim of the present study was twofold. First, we investigated the associations of four SNPs (i.e., $A L D H 2$ rs671, $A D H 1 B$ rs1229984, $A D H 1 B$ rs1042026, and $A D H 1 C$ rs1693482) and cognitive function among nearly 2,000 Chinese oldestold from a population-based cohort study. Considering the high prevalence of $A D H$ and $A L D H$ gene mutation in the Chinese population and their unique characterization, we then investigated whether this association would be modified by the level of alcohol consumption.

\section{MATERIALS AND METHODS}

\section{Participants}

The present study used data from the CLHLS, which is an ongoing longitudinal study that began in 1998 with follow-up surveys for every 2-3 years. The CLHLS is a Chinese nationwide survey conducted in randomly selected counties and cities in 22 of 30 provinces covering $85 \%$ of the population of China. All centenarians from the selected areas who agreed to participate were included in the study. Based on sex and place of residence (i.e., living in the same street, village, city, or county) for a given centenarian, randomly selected octogenarians and nonagenarians were also sampled. More details about the sampling procedure and quality of data of this survey have been published elsewhere (Zeng et al., 2017). Ethical approval was obtained from the Research Ethics Committees of Peking University and Duke University (IRB00001052-13074). All participants or their legal representatives signed written consent forms in the baseline and follow-up surveys. In this cross-sectional study, we derived the data from the baseline survey of each participant. The analyses were based on the CLHLS genetic dataset, comprising 1,949 adults aged over 90 years.

\section{Cognitive Function}

The cognitive function of CLHLS participants was assessed by the Chinese version of the Mini-Mental State Examination (MMSE) through a home-based interview, which includes 24 items, covering 7 subscales including orientation (4 points for time orientation and 1 point for place orientation); naming foods (naming as many kinds of food as possible in $1 \mathrm{~min}$, 7 points); registration of 3 words (3 points); attention and calculation (mentally subtracting 3 iteratively from 20, 5 points); copy a figure (1 point); recall (delayed recall of the 3 words mentioned above, 3 points); and language ( 2 points for naming objectives, 1 point for repeating a sentence, and 3 points for listening and following directions). The MMSE score ranges from 0 to 30 . Higher scores represent a better cognitive function. The validity and reliability of this Chinese MMSE have been verified in several previous studies (Zhang, 2006; An and Liu, 2016). Consistent with previous studies (Zhang et al., 2019), because a high proportion of our participants did not have formal education $(\sim 70 \%)$, cognitive impairment was defined as an MMSE score of $<18$. 


\section{Alcohol Consumption and Other Covariates}

During each interview, the interviewers measured a range of demographic, behavioral, and socioeconomic covariates. Alcohol consumption was self-reported and categorized as heavy, moderate, or never drinkers. Current alcohol users who consumed $>25 \mathrm{~g}$ of alcohol per day (for men) and $>15 \mathrm{~g}$ (for women) were considered heavy drinkers. Current alcohol users who consumed $\leq 25 \mathrm{~g}$ (for men) and $\leq 15 \mathrm{~g}$ (for women) were considered moderate drinkers. Participants who reported they never drank were considered never drinkers. All variables were measured at baseline (indicate year). All selfreported information was collected through face-to-face home interview by trained research staff members with more than 12 years of education.

Following the previous studies, we included the following variables as confounders: age (years), sex, residence (rural vs. urban), education years, smoking (never, former, and current), regular physical activity (yes vs. no), dietary pattern, leisure activity score, and self-reported chronic diseases [e.g., hypertension, diabetes, heart disease, stroke, and chronic obstructive pulmonary disease (COPD)]. Dietary pattern was categorized as unfavorable, intermediate, or favorable by a simplified healthy eating index based on the intake frequency of nine food categories, including fruits, vegetables, fish, bean products, tea, garlic, eggs, sugar, and salt-preserved vegetables. A 3-point scale question was used to measure the current intake frequency of each food group: "always or almost every day," "sometimes or occasionally," or "rarely or never." Those 3 terms received the scores of 2,1 , or 0 , respectively, with higher scores indicating a higher level of consumption. Two of those food groups, i.e., sugar and salt-preserved vegetables, were received the scores of 0,1 , and 2, respectively, for some evidence of the negative impact of the high consumption of those two food groups. The leisure activity score was summarized from the following eight activities: visiting neighbors, shopping, cooking, washing clothes, walking $1 \mathrm{~km}$, lifting $5 \mathrm{~kg}$, crouching and standing up three times, and taking public transportation. We scored each activity as 1 for "never," 2 for "sometimes," and 3 for "almost every day." The score ranged from 5 to 21 with a higher score, indicating more leisure activities. All self-reported information was collected through face-to-face home interview by trained research staff members at the baseline survey. Interviewees were encouraged to answer as many questions as possible. If they were unable to answer questions, a close family member or another proxy, such as a primary caregiver, provided answers (Zeng, 2012).

\section{Genotyping}

The CLHLS collected DNA samples from parts of participants in 1998, 2000, 2002, 2005, 2008-2009, and 2011-2012 waves of the survey. Genotyping of DNA samples was produced by the Beijing Genomics Institute (BGI), and the BGI genotyping quality control procedures of the CLHLS genetic study have been published elsewhere (Zeng et al., 2016). We extracted four SNPs associated with alcohol metabolism from the GWAS data (i.e., $A L D H 2$ rs671, $A D H 1 B$ rs1229984, $A D H 1 B$ rs1042026, and $A D H 1 C$ rs1693482).

The genotypes were defined by following the additive and dominant models. In a dominant model, any genotype that contains one or two copies of the minor allele is coded as one; otherwise, the genotype that does not contain any copy of the minor allele is coded as zero. In an additive model, carrying two copies of the minor allele was coded as three. One copy of the minor allele with one copy of the major allele and two major alleles were coded as two and one, respectively. We mainly used the dominant models in the GxE analysis to define the genotype because it can distinguish the genotype carriers and non-carriers, but the additive model cannot. In addition, further grouping the samples in the additive model would result in many more GxE interaction terms in the regressions and would, in turn, negatively affect the estimates and complicate the discussions.

\section{Statistical Analysis}

The characteristics of participants were compared according to cognitive function (with vs. without impairment). Means and standard deviations (SDs) were calculated for continuous variables; counts and percentages were calculated for categorical variables. We used both logistic regression (binary outcome: cognitive impairment) and linear regression (continuous outcome: MMSE score) models to examine the unadjusted and adjusted associations between the four polymorphisms and cognitive impairment, respectively. We build two regression models, namely, partly adjusted: adjusted for age at baseline in years, sex, residency, and education years; and fully adjusted: additionally adjusted for smoking status, alcohol consumption, current physical activity, dietary pattern and leisure activity score, and five kinds of self-reported diseases on the basement of the partly adjusted model.

To examine whether the association between the four polymorphisms and cognitive impairment was modified by alcohol consumption, we first ran a logistic regression model with an interaction term between those four polymorphisms and alcohol consumption, respectively, and then conducted the regression analysis among persons with different levels of alcohol consumption (e.g., heavy, moderate, and never drinkers), separately.

All statistical analyses were conducted using STATA version 14.0 (Stata Corp., College Station, TX, United States).

\section{RESULTS}

\section{Study Sample Characteristics}

Demographic and the four polymorphisms information are detailed by cognitive function in Table 1. A total of 1,949 participants were included after excluding 101 participants who lacked cognitive assessment $(n=64)$ and aged below 90 years $(n=37)$. Of 1,949 participants aged over 90 years, the average age was $101.3 \pm 3.3$ years; $76.3 \%$ were women. Participants with cognitive impairment are more likely to be older, women, with lower education level, not smoking, with unfavorable 
TABLE 1 | Selected characteristics of the participants by cognitive function.

\begin{tabular}{|c|c|c|c|c|}
\hline \multirow[t]{2}{*}{ Characteristics $^{\mathrm{a}}$} & \multirow[b]{2}{*}{$\begin{array}{c}\text { Total } \\
N=1,949\end{array}$} & \multicolumn{2}{|c|}{ Cognitive impairment ${ }^{a}$} & \multirow[t]{2}{*}{$P$-value } \\
\hline & & $\begin{array}{l}\text { Without } \\
N=805\end{array}$ & $\begin{array}{c}\text { With } \\
N=1,144\end{array}$ & \\
\hline \multicolumn{5}{|c|}{ Count (\%) unless otherwise indicated } \\
\hline Age, mean \pm SD & $101.3(3.3)$ & $100.9(3.3)$ & $101.5(3.2)$ & $<0.001$ \\
\hline Sex & & & & $<0.001$ \\
\hline Women & $1487(76.3)$ & $540(67.1)$ & $947(82.8)$ & \\
\hline Men & $462(23.7)$ & $265(32.9)$ & $197(17.2)$ & \\
\hline $\begin{array}{l}\text { MMSE score, } \\
\text { mean } \pm S D\end{array}$ & $14.2(10.4)$ & $24.9(3.4)$ & $6.76(6.4)$ & $<0.001$ \\
\hline Residence & & & & 0.081 \\
\hline Urban & $719(36.9)$ & $323(40.1)$ & $396(34.6)$ & \\
\hline Rural & $1230(63.1)$ & $482(59.9)$ & $748(65.4)$ & \\
\hline $\begin{array}{l}\text { Education years, } \\
\text { mean } \pm \text { SD }\end{array}$ & $0.93(2.5)$ & $1.5(3.2)$ & $0.57(1.8)$ & $<0.001$ \\
\hline Alcohol consumption & & & & 0.053 \\
\hline Never drink & $1513(77.6)$ & $604(75.0)$ & $909(79.5)$ & \\
\hline Moderate drink & $199(10.2)$ & $88(10.9)$ & $111(9.7)$ & \\
\hline Heavy drink & 237 (12.2) & $113(14.0)$ & $124(10.8)$ & \\
\hline Smoking & & & & 0.026 \\
\hline Never & $1712(90.6)$ & $828(89.0)$ & $884(92.2)$ & \\
\hline Former & $148(7.8)$ & $82(8.8)$ & $66(6.9)$ & \\
\hline Current & $29(1.5)$ & $20(2.2)$ & $9(0.9)$ & \\
\hline Dietary pattern ${ }^{\mathbf{b}}$ & & & & $<0.001$ \\
\hline Unfavorable & $804(41.3)$ & $261(32.4)$ & $543(47.5)$ & \\
\hline Intermediate & 634 (32.5) & $273(33.9)$ & 361 (31.6) & \\
\hline Favorable & $511(26.2)$ & $271(33.7)$ & $240(21.0)$ & \\
\hline $\begin{array}{l}\text { Regular physical } \\
\text { activity }\end{array}$ & & & & 0.05 \\
\hline Yes & $522(27.8)$ & $310(33.4)$ & $212(22.2)$ & \\
\hline No & $1359(72.2)$ & $617(66.6)$ & $742(77.8)$ & \\
\hline Leisure activity score ${ }^{b}$ & $9.6(2.4)$ & $10.6(2.5)$ & $8.7(1.9)$ & $<0.001$ \\
\hline$A L D H 2, \mathrm{rs} 671^{\mathrm{c}}$ & & & & 0.083 \\
\hline$G G$ & $527(65.5)$ & $733(64.1)$ & $1260(64.6)$ & \\
\hline$A G$ & 241 (29.9) & $359(31.4)$ & $600(30.8)$ & \\
\hline AA & $37(4.6)$ & $52(4.5)$ & $89(4.6)$ & \\
\hline$A D H 1 B$, rs1229984 & & & & 0.315 \\
\hline$\pi$ & $495(61.5)$ & 639 (55.9) & 1134 (58.2) & \\
\hline CT & 254 (31.6) & $431(37.7)$ & $685(35.1)$ & \\
\hline $\mathrm{CC}$ & $56(7.0)$ & $74(6.5)$ & $130(6.7)$ & \\
\hline$A D H 1 B$, rs 1042026 & & & & 0.328 \\
\hline $\mathrm{CC}$ & $516(64.1)$ & $683(59.7)$ & 1199 (61.5) & \\
\hline СT & 244 (30.3) & $398(34.8)$ & 642 (32.9) & \\
\hline$\pi$ & $45(5.6)$ & $63(5.5)$ & $108(5.5)$ & \\
\hline$A D H 1 C$, rs1693482 & & & & 0.323 \\
\hline $\mathrm{CC}$ & 694 (86.2) & $958(83.7)$ & $1652(84.8)$ & \\
\hline СТ & $103(12.8)$ & $177(15.5)$ & $280(14.4)$ & \\
\hline$\pi$ & $8(1.0)$ & $9(0.8)$ & $17(0.9)$ & \\
\hline
\end{tabular}

MMSE, Mini-Mental State Examination.

${ }^{a}$ Cognitive impairment: cognitive impairment was defined by an MMSE score of $<18$.

${ }^{b}$ Dietary score: the score was calculated from the frequency for intake of nine foods: fruits, vegetables, fish, bean products, tea, garlic, eggs, sugar, and salt-preserved vegetables. For two of 9 variables, i.e., sugar and saltpreserved vegetables, the answer of "always or almost every day," "sometimes or occasionally," or "rarely or never" received the scores of 0, 1, or 2, respectively; for the other 7 variables, the same three responses received the scores of 2 , 1 , or 0 , respectively. Scores for the 9 variables were then summed to obtain a scale ranging from 0 to 18 with higher scores indicating higher frequency for fruits, vegetables, fish, bean products, tea, garlic, and eggs, while higher scores meant lower frequencies for sugar and salt-preserved vegetables. The dietary pattern was defined by the trisection of the dietary pattern score (lowest trisection: unfavorable, intermediate: intermediate, highest: favorable).

'Leisure activity score: eight activities were assessed: visiting neighbors, shopping, cooking, washing clothes, walking $1 \mathrm{~km}$, lifting $5 \mathrm{~kg}$, crouching and standing up three times, and taking public transportation. We scored each activity as 1 for "never," 2 for "sometimes," and 3 for "almost every day." The score ranged from 5 to 21 with higher score indicating more leisure activities. dietary pattern, and with lower leisure activities (Table 1, $p$-values $<0.05)$.

\section{Association of Cognitive Function With $A D H$ and $A L D H 2$ Genes}

In the partially adjusted logistic regression model, carrying $\mathrm{A}$ allele on rs671, C allele on rs1229984, T allele on rs1042026, and $\mathrm{T}$ allele on rs1693482 was associated with higher odds of cognitive impairment [rs671: odds ratio (OR): 1.19, 95\% CI: 0.97, 1.47; rs1229984: OR: 1.27, 95\% CI: 1.00, 1.61; rs1042026: OR: 1.26, 95\% CI: 1.04, 1.55; rs1693482: OR: 1.19, 95\% CI: 0.91, 1.57; Table 2]. In the fully adjusted logistic regression model, the associations of rs671, rs1229984, and rs1042026 with cognitive impairment persisted to be significant. In the partially adjusted linear regression model, carrying $\mathrm{T}$ allele on rs1042026 and T allele on rs1693482 was significantly associated with a lower MMSE score of 0.90 (95\% CI: 0.01, 1.80). In the fully adjusted linear regression model, carrying A on rs671 was associated with a lower MMSE score of 1.05 (95\% CI: -2.03, -0.09).

In the additive model, the one major allele with one minor allele genotypes of rs671, rs1229984, and rs1042026 was significantly associated with lower MMSE scores and higher odds of cognitive impairment compared with the two major allele genotypes (Table 2). However, the two minor allele genotypes of those four SNPs were not significantly associated with MMSE score or cognitive impairment in the fully or partially adjusted model.

\section{Association Between Alcohol Consumption and Cognitive Function}

The association between alcohol consumption and cognitive impairment was analyzed in the partially adjusted model and fully adjusted model (Supplementary Table 1). In the fully adjusted linear regression model, moderate alcohol use and heavy alcohol use were significantly associated with a lower MMSE score (moderate: $-2.52,95 \%$ CI: $-2.49,-2.55$; heavy: -2.83 , 95\% CI: $-2.36,-3.30)$ compared with participants who never consumed alcohol.

\section{Subgroup Analyses by Alcohol Consumption}

Table 3 presents the associations between four SNPs (those specified here) with the dominant model and cognitive impairment stratified by alcohol consumption. In the subgroup analyses, there is no evidence that the association of each of four SNPs with cognitive impairment differed by alcohol consumption ( $p$-values for interactions $>0.05)$.

\section{DISCUSSION}

Using data from nearly 2,000 Chinese adults aged over 90 years, we found that the SNPs on $A D H$ and $A L D H 2$ genes were associated with higher odds of cognitive impairment. In addition, the results showed that the associations between those polymorphisms and cognitive impairments were not modified by 
TABLE 2 | Association of ALDH2 rs671, ADH1B rs1229984, ADH1B rs1042026, and ADH1C rs1693482 polymorphisms with cognitive impairment. ${ }^{a}$

\begin{tabular}{|c|c|c|c|c|}
\hline \multirow[t]{2}{*}{ Independent variable } & \multicolumn{2}{|c|}{$\begin{array}{c}\text { Logistic regression } \\
\text { OR of cognitive impairment }{ }^{\mathrm{a}},(95 \% \mathrm{Cl})\end{array}$} & \multicolumn{2}{|c|}{$\begin{array}{c}\text { Linear regression } \\
\text { Coefficient for MMSE score }(95 \% \mathrm{Cl})\end{array}$} \\
\hline & Partially adjusted ${ }^{b}$ & Fully adjusted $^{c}$ & Partially adjusted & Fully adjusted ** \\
\hline \multicolumn{5}{|l|}{ Additive model } \\
\hline rs671 GG vs. AG & $1.19(1.00,1.47)^{\star \star}$ & $1.36(1.07,1.74)^{\star \star}$ & $-0.61(-1.62,0.40)$ & $-1.23(-2.22,-0.26)$ ** \\
\hline rs671 GG vs. AA & $1.02(0.64,1.64)$ & $1.12(0.66,1.90)$ & $1.25(-0.99,3.51)$ & $0.84(-1.32,3.00)$ \\
\hline rs1229984 TT vs. CT & $1.30(1.06,1.60)$ ** & $1.32(1.05,1.67)$ ** & $-1.11(-2.09,-0.13)$ ** & $-1.02(-1.97,-0.08)^{\star *}$ \\
\hline rs1229984 TT vs. CC & $1.25(0.83,1.88)$ & $1.09(0.70,1.72)$ & $-0.17(-2.13,1.78)$ & $0.35(-1.49,2.21)$ \\
\hline rs1042026 CC vs. CT & $1.22(1.00,1.51)^{\star \star}$ & $1.29(1.03,1.64)^{\star *}$ & $-0.82(-1.82,0.17)^{*}$ & $-0.92(-1.87,-0.01)^{\star *}$ \\
\hline rs1042026 CC vs. TT & $1.29(0.83,2.03)$ & $1.05(0.65,1.72)$ & $0.02(-2.11,2.16)$ & $0.99(-1.04,3.04)$ \\
\hline rs1693482 CC vs. CT & $1.22(0.93,1.62)$ * & $1.16(0.84,1.59)$ & $-1.39(-2.71,-0.08)^{\star *}$ & $-1.27(-2.55,-0.004)^{* *}$ \\
\hline rs1693482 CC vs. TT & $0.88(0.31,2.56)$ & $1.07(0.34,3.28)$ & $2.54(-2.58,7.68)$ & $1.39(-3.50,6.28)$ \\
\hline \multicolumn{5}{|l|}{ Dominant model } \\
\hline rs671 GG vs. AG/AA & $1.19(0.97,1.47)^{*}$ & $1.33(1.05,1.69) * *$ & $-0.53(-1.49,0.42)$ * & $-1.05(-2.03,-0.09)$ ** \\
\hline rs1229984 TT vs. CT/CC & $1.27(1.00,1.61)$ ** & $1.33(1.02,1.75)$ ** & $-0.90(-1.80,-0.01)^{* *}$ & $-0.93(-2.00,0.12)^{*}$ \\
\hline rs1042026 CC vs. CT/TT & $1.26(1.04,1.55)$ ** & $1.29(1.03,1.62) * *$ & $-0.76(-1.69,0.17)$ & $-0.70(-1.63,0.23)$ \\
\hline rs1693482 CC vs. CT/TT & $1.19(0.91,1.57)^{*}$ & $1.15(0.84,1.57)$ & $-1.19(-2.42,0.05)^{*}$ & $-1.09(-2.35,0.15)^{*}$ \\
\hline
\end{tabular}

alcohol consumption, which is different from the findings of a recent experimental study (Joshi et al., 2019).

The results indicated that carrying the $A L D H 2$ and $A D H$ mutation was associated with higher odds of cognitive impairment among the oldest-old population. Its mutation results in a reduction of the $A L D H 2$ enzymatic activity, which is widely mutated in the Asian population $(\sim 30 \%)$. One possible explanation was that those two mutations have a negative impact on mitochondrial functions and the metabolism of 4-hydroxy-2-nonenal (4-NHE). ALDH2 and $A D H$ are involved in the detoxification of the ethanol metabolism and other

TABLE 3 | The association of ALDH2 rs671 polymorphism with cognitive impairment stratified by alcohol consumption and fresh fruit consumption. ${ }^{a}$

\begin{tabular}{|c|c|c|c|}
\hline & $\begin{array}{l}\text { Participants } \\
\text { (N) }\end{array}$ & $\begin{array}{c}\text { Odds ratio } \\
(95 \% \text { Confidence Interval })^{b}\end{array}$ & $\begin{array}{c}P \text { for } \\
\text { interaction }\end{array}$ \\
\hline \multicolumn{4}{|l|}{ Alcohol consumption } \\
\hline \multicolumn{4}{|l|}{ Never } \\
\hline rs671 GG vs. AG/AA & $912 / 601$ & $1.25(0.97,1.62)$ & \\
\hline rs1229984 TT vs. CT/CC & $889 / 624$ & $1.36(1.05,1.75)$ & \\
\hline rs1042026 CC vs. CT/TT & $937 / 576$ & $1.38(1.07,1.79)$ & \\
\hline rs1693482 CC vs. CT/TT & $1295 / 218$ & $1.39(0.95,2.03)$ & \\
\hline \multicolumn{4}{|l|}{ Moderate drink } \\
\hline rs671 GG vs. AG/AA & $146 / 53$ & $1.45(0.63,3.34)$ & 0.58 \\
\hline rs1229984 TT vs. CT/CC & $121 / 78$ & $0.97(0.47,2.02)$ & 0.44 \\
\hline rs1042026 CC vs. CT/TT & $131 / 68$ & $0.77(0.36,1.63)$ & 0.15 \\
\hline rs1693482 CC vs. CT/TT & $167 / 32$ & $0.47(0.19,1.23)$ & 0.079 \\
\hline \multicolumn{4}{|l|}{ Heavy drink } \\
\hline rs671 GG vs. AG/AA & $203 / 34$ & $2.14(0.84,5.50)$ & 0.26 \\
\hline rs1229984 TT vs. CT/CC & $124 / 113$ & $1.13(0.63,2.13)$ & 0.63 \\
\hline rs1042026 CC vs. CT/TT & $131 / 106$ & $1.01(0.54,1.90)$ & 0.51 \\
\hline rs1693482 CC vs. CT/TT & $190 / 47$ & $0.85(0.39,1.87)$ & 0.54 \\
\hline
\end{tabular}

aldehydes, including 4-HNE. It has been shown that $4 \mathrm{HNE}$ concentration increased in the brain tissue of the $A L D H 2^{*} 2$ transgenic mice in an age-dependent manner. Such increases correlated with neurodegeneration memory loss and $\mathrm{AD}$ like pathological changes in these $A L D H 2^{*} 2$ transgenic mice (Ohsawa et al., 2008). In addition, $4 \mathrm{HNE}$ levels are higher in the hippocampus of postmortem samples from patients with $A D$ (Williams et al., 2006). Among the population level, previous studies reported the inconsistent results on this association. A cross-sectional case-control study has shown that ALDH2 genotype is associated with cognitive function among 139 Chinese patients with Parkinson's disease (mean age: 63.0 years) (Yu et al., 2016), and another study has demonstrated its association among 411 Chinese with an average age of 77.4 years (Wang et al., 2008). However, in another study including 690 Korean community residents (mean age: 72.8 years) (Kim et al., 2004), the association between $A L D H 2$ genotype and cognitive functions was not significant. This paradox finding may be due to the mean age difference of study samples. It is plausible that the genetic risk of carrying the $A L D H 2$ mutation is cumulative (Licher et al., 2019). Researchers found that $A P O E$ as well as other common genetic variants could have a cumulative risk on the progression of dementia and $\mathrm{AD}$ as age advances (van der Lee et al., 2018). To be more specific, with the negative influence of those mutations on neuronal functioning, the carriers may have a higher speed of neuronal cell loss which is irreversible. Accordingly, during the early life stage, the difference in cognitive function between the carriers and non-carriers may not be significant, while as people age, the harmful effects of genetic variants may build up, and the carriers may have a lower average cognitive function compared with non-carriers of the same age due to the cumulative damage on neurons (Bai and Mei, 2011). In addition to rs671 on ALDH2, we also found that rs1229984 and rs1042026 on $A D H 1 B$ gene were associated with the cognitive function among the oldest-old 
population. The $A D H 1 B$ rs1229984 $\mathrm{T}$ allele encodes a super active enzyme subunit that could accelerate the conversion from ethanol into acetaldehyde (Lin et al., 2021). ADH1C rs1693482 is found to be associated with a twofold difference in $A D H \mathrm{Vmax}$ in vitro (Birley et al., 2009). One experimental study has found that in the animal model of $\mathrm{AD}$, the overexpressing of $A D H 1 B$ suppresses the $\beta$-amyloid-induced neuron apoptosis (Wang et al., 2019). Corresponding the results, it may indicate that the genes associated with alcohol metabolism might be important in the progress of cognitive impairment or $\mathrm{AD}$. To note, these results also determined the potential role of mitochondrial dysfunctions in the pathology of $\mathrm{AD}$.

We found that those associations were not modified by alcohol consumption. One experimental study found that the ALDH2*2 mutation increases the damage by sustained ethanol exposure in mouse brain, and they found that $A L D H 2 * 2$ deficiency increases ethanol-induced neuroinflammation in vivo (Joshi et al., 2019). However, we found that on the population level, such interactive effect was not significant. It may indicate that the damage of alcohol to the brain is stable and consistent which may not be varied by genotype. In addition, another possible explanation was that the consumption of stored fruits and vegetables, as well as fruit juice, would bring ethanol into the human body and the total ethanol intake is more difficult to measure.

Methodological strengths of this study include a large sample size of the oldest old, and we have included multiple genes related to alcohol metabolism. This study also has several limitations: (1) it has a cross-sectional design and cannot evaluate changes in alcohol consumption or establish causality. However, the results were robust to adjustment of a number of indicators such as diseases. Nevertheless, prospective studies on the incidence of cognitive impairment are warranted; (2) the alcohol consumption was self-reported, thus non-specific (Muntner et al., 2014). This possibility of recall bias is a common concern in the longitudinal cohort studies, although CLHLS data have been validated as being reliable in previous studies (Zeng et al., 2008). In the CLHLS study, all self-reported information was collected through face-to-face home interview by trained research staff members. Interviewees were encouraged to answer as many questions as possible. If they were unable to answer questions, a close family member or another proxy, such as a primary caregiver, provided answers (Zeng et al., 2008). (3) We used the Chinese version of MMSE to measure cognition, which is not a clinical diagnosis for cognitive impairment (Zhang, 1993; Chou, 2003). However, it is a validated instrument in population-based studies. (4) As the study included only participants who aged over 90 years and the average lifespan of women is longer than men, a higher proportion of women were taken into the analysis sample which may lead to bias. (5) The percentage of drinker is $20 \%$ and the results need to be validated within a larger cohort.

\section{REFERENCES}

An, R., and Liu, G. G. (2016). Cognitive impairment and mortality among the oldest-old Chinese. Int. J. Geriatr. Psychiatry 31, 1345-1353. doi: 10.1002/gps. 4442

Au Yeung, S. L., Leung, G. M., Chan, W. M., Hui, Y. F., Lam, T. H., Schooling, C. M., et al. (2011). Moderate alcohol use and cognitive function in an elderly

\section{CONCLUSION}

We found that some SNPs associated with alcohol metabolism were associated with higher odds of cognitive impairment among the Chinese oldest-old and those associations were independent of alcohol consumption. Due to its mutation can be easily observed even without genotyping, it may have more public health implications especially for the Asian population on the AD risk stratification and alcohol control. Further studies on how to modify the genetic risk for cognitive impairment associated with gene mutation associated with alcohol metabolism are warranted.

\section{DATA AVAILABILITY STATEMENT}

Publicly available datasets were analyzed in this study. This data can be found here: https://sites.duke.edu/centerforaging/ programs/chinese-longitudinal-healthy-longevity-survey-clhls.

\section{ETHICS STATEMENT}

The studies involving human participants were reviewed and approved by the Biomedical Ethics Review Committee of Peking University (IRB00001052-11015). The patients/participants provided their written informed consent to participate in this study.

\section{AUTHOR CONTRIBUTIONS}

All authors listed have made a substantial, direct, and intellectual contribution to the work, and approved it for publication.

\section{FUNDING}

This study was supported by the National Natural Science Foundation of China (71110107025, 71233001, and 71490732) to YZ, the National Institute on Aging/National Institutes of Health (R01 AG023627) to YZ, and 2020YFC2005600 from the Chinese Ministry of Science and Technology to CW.

\section{SUPPLEMENTARY MATERIAL}

The Supplementary Material for this article can be found online at: https://www.frontiersin.org/articles/10.3389/fnagi. 2021.710966/full\#supplementary-material

Chinese cohort. J. Am. Geriatr. Soc. 59, 172-174. doi: 10.1111/j.1532-5415.2010. 03195.x

Bai, J., and Mei, Y. (2011). Overexpression of aldehyde dehydrogenase-2 attenuates neurotoxicity induced by 4-hydroxynonenal in cultured primary hippocampal neurons. Neurotox. Res. 19, 412-422. doi: 10.1007/s12640-010-9183-1

Birley, A. J., James, M. R., Dickson, P. A., Montgomery, G. W., Heath, A. C., Martin, N. G., et al. (2009). ADH single nucleotide polymorphism associations with 
alcohol metabolism in vivo. Hum. Mol. Genet. 18, 1533-1542. doi: 10.1093/ $\mathrm{hmg} / \mathrm{ddp} 060$

Bray, N. J., Preece, A., Williams, N. M., Moskvina, V., Buckland, P. R., Owen, M. J., et al. (2005). Haplotypes at the dystrobrevin binding protein 1 (DTNBP1) gene locus mediate risk for schizophrenia through reduced DTNBP1 expression. Hum. Mol. Genet. 14, 1947-1954. doi: 10.1093/hmg/ddi199

Chen, C. H., Ferreira, J. C., Gross, E. R., and Mochly-Rosen, D. (2014). Targeting aldehyde dehydrogenase 2: new therapeutic opportunities. Physiol. Rev. 94, 1-34. doi: 10.1152/physrev.00017.2013

Chen, X., Guo, C., and Kong, J. (2012). Oxidative stress in neurodegenerative diseases. Neural. Regen. Res. 7, 376-385. doi: 10.3969/j.issn.16735374.2012.05.009

Chou, K.-L. (2003). Correlates of everyday competence in Chinese older adults. Aging Ment. Health 7, 308-315.

Eng, M. Y., Luczak, S. E., and Wall, T. L. (2007). ALDH2, ADH1B, and ADH1C genotypes in Asians: a literature review. Alcohol Res. Health 30, 22-27.

Hurley, T. D., and Edenberg, H. J. (2012). Genes encoding enzymes involved in ethanol metabolism. Alcohol Res. 34, 339-344.

Joshi, A. U., Van Wassenhove, L. D., Logas, K. R., Minhas, P. S., Andreasson, K. I., Weinberg, K. I., et al. (2019). Aldehyde dehydrogenase 2 activity and aldehydic load contribute to neuroinflammation and Alzheimer's disease related pathology. Acta Neuropathol. Commun. 7:190. doi: 10.1186/s40478-0190839-7

Kamino, K., Nagasaka, K., Imagawa, M., Yamamoto, H., Yoneda, H., Ueki, A., et al. (2000). Deficiency in mitochondrial aldehyde dehydrogenase increases the risk for late-onset Alzheimer's disease in the Japanese population. Biochem. Biophys. Res. Commun. 273, 192-196. doi: 10.1006/bbrc.2000.2923

Kim, J. M., Stewart, R., Shin, I. S., Jung, J. S., and Yoon, J. S. (2004). Assessment of association between mitochondrial aldehyde dehydrogenase polymorphism and Alzheimer's disease in an older Korean population. Neurobiol. Aging 25, 295-301. doi: 10.1016/S0197-4580(03)00114-3

Koch, M., Fitzpatrick, A. L., Rapp, S. R., Nahin, R. L., Williamson, J. D., Lopez, O. L., et al. (2019). Alcohol Consumption and Risk of Dementia and Cognitive Decline Among Older Adults With or Without Mild Cognitive Impairment. JAMA Netw. Open 2:e1910319. doi: 10.1001/jamanetworkopen.2019.10319

Komulainen, P., Pedersen, M., Hänninen, T., Bruunsgaard, H., Lakka, T. A., Kivipelto, M., et al. (2008). BDNF is a novel marker of cognitive function in ageing women: the DR's EXTRA Study. Neurobiol. Learn. Mem. 90, 596-603. doi: 10.1016/j.nlm.2008.07.014

Licher, S., Ahmad, S., Karamujic-Comic, H., Voortman, T., Leening, M. J. G., Ikram, M. A., et al. (2019). Genetic predisposition, modifiable-risk-factor profile and long-term dementia risk in the general population. Nat. Med. 25, 13641369. doi: 10.1038/s41591-019-0547-7

Lin, C. H., Nfor, O. N., Ho, C. C., Hsu, S. Y., Tantoh, D. M., Liaw, Y. C., et al. (2021). Association of ADH1B polymorphism and alcohol consumption with increased risk of intracerebral hemorrhagic stroke. J. Transl. Med. 19:227. doi: 10.1186/s12967-021-02904-4

Macgregor, S., Lind, P. A., Bucholz, K. K., Hansell, N. K., Madden, P. A., Richter, M. M., et al. (2009). Associations of ADH and ALDH2 gene variation with self report alcohol reactions, consumption and dependence: an integrated analysis. Hum. Mol. Genet. 18, 580-593. doi: 10.1093/hmg/ddn372

Muntner, P., Colantonio, L. D., Cushman, M., Colantonio, L. D., Cushman, M., Goff, D. C. Jr., et al. (2014). Validation of the atherosclerotic cardiovascular disease Pooled Cohort risk equations. JAMA 311, 1406-1415. doi: 10.1001/ jama.2014.2630

Ohsawa, I., Nishimaki, K., Murakami, Y., Suzuki, Y., Ishikawa, M., Ohta, S., et al. (2008). Age-dependent neurodegeneration accompanying memory loss in transgenic mice defective in mitochondrial aldehyde dehydrogenase 2 activity. J. Neurosci. 24, 6239-6249. doi: 10.1523/JNEUROSCI.4956-07.2008

Swerdlow, R. H. (2018). Mitochondria and Mitochondrial Cascades in Alzheimer's Disease. J. Alzheimer's Dis. 62, 1403-1416. doi: 10.3233/JAD-170585

Topiwala, A., and Ebmeier, K. P. (2018). Effects of drinking on late-life brain and cognition. Evid. Based Ment. Health 21, 12-15. doi: 10.1136/eb-2017-10 2820

van der Lee, S. J., Wolters, F. J., Ikram, M. K., Hofman, A., Ikram, M. A., Amin, N., et al. (2018). The effect of APOE and other common genetic variants on the onset of Alzheimer's disease and dementia. Lancet Neurol. 17, 434-444. doi: 10.1016/S1474-4422(18)30053-X

Wang, B., Wang, J., Zhou, S., Tan, S., He, X., Yang, Z., et al. (2008). The association of mitochondrial aldehyde dehydrogenase gene (ALDH2) polymorphism with susceptibility to late-onset Alzheimer's disease in Chinese. J. Neurol. Sci. 268, 172-175. doi: 10.1016/j.jns.2007.12.006

Wang, Y., Zhang, Y., Zhang, X., Yang, T., Liu, C., and Wang, P. (2019). Alcohol Dehydrogenase 1B Suppresses $\beta$-Amyloid-Induced Neuron Apoptosis. Front. Aging Neurosci. 11:135. doi: 10.3389/fnagi.2019.00135

Williams, T. I., Lynn, B. C., Markesbery, W. R., and Lovell, M. A. (2006). Increased levels of 4-hydroxynonenal and acrolein, neurotoxic markers of lipid peroxidation, in the brain in Mild Cognitive Impairment and early Alzheimer's disease. Neurobiol. Aging 27, 1094-1099. doi: 10.1016/j.neurobiolaging.2005. 06.004

Wray, N. R., James, M. R., Handoko, H. Y., Dumenil, T., Lind, P. A., Montgomery, G. W., et al. (2008). Association study of candidate variants from brain-derived neurotrophic factor and dystrobrevin-binding protein 1 with neuroticism, anxiety, and depression. Psychiatric Genet. 18, 219-225. doi: 10.1097/YPG. 0b013e3283050aee

Wu, Y. Y., Lee, Y. S., Liu, Y. L., Hsu, W. C., Ho, W. M., Huang, Y. H., et al. (2021). Association Study of Alcohol Dehydrogenase and Aldehyde Dehydrogenase Polymorphism With Alzheimer Disease in the Taiwanese Population. Front. Neurosci. 15:625885. doi: 10.3389/fnins.2021. 625885

Yu, R. L., Tan, C. H., Lu, Y. C., and Wu, R. M. (2016). Aldehyde dehydrogenase 2 is associated with cognitive functions in patients with Parkinson's disease. Sci. Rep. 6:30424. doi: 10.1038/srep30424

Zeng, Y. (2012). Towards Deeper Research and Better Policy for Healthy Aging -Using the Unique Data of Chinese Longitudinal Healthy Longevity Survey. China Eco. J. 5, 131-149. doi: 10.1080/17538963.2013.76 4677

Zeng, Y., Feng, Q., Gu, D., and Vaupel, J. W. (2017). Demographics, phenotypic health characteristics and genetic analysis of centenarians in China. Mech. Ageing Dev. 165, 86-97. doi: 10.1016/j.mad.2016.12.010

Zeng, Y., Nie, C., Min, J., Liu, X., Li, M., Chen, H., et al. (2016). Novel loci and pathways significantly associated with longevity. Sci. Rep. 6:21243. doi: $10.1038 /$ srep 21243

Zeng, Y., Poston, D. L., Vlosky, D. A., and Gu, D. (2008). "An exploration of the subjective well-being of the Chinese oldest-old," in Healthy Longevity in China: Demographic, Socioeconomic, and Psychological Dimensions, eds Y. Zeng, D. L. Poston Jr., D. A. Vlosky, and D. Gu (Springer: Business Media), 347-356. doi: 10.1007/978-1-4020-6752-5_21

Zhang, M. Y. (1993). Manual of Evaluation of Scales in Psychiatrics. Changsha: Hunan Science and Technology Press, 38-41.

Zhang, Q., Wu, Y., Han, T., and Liu, E. (2019). Changes in Cognitive Function and Risk Factors for Cognitive Impairment of the Elderly in China: 20052014. Int. J. Environ. Res. Public Health 16:2847. doi: 10.3390/ijerph1616 2847

Zhang, Y., and Ren, J. (2011). ALDH2 in alcoholic heart diseases: molecular mechanism and clinical implications. Pharmacol. Ther. 132, 86-95. doi: 10. 1016/j.pharmthera.2011.05.008

Zhang, Z. (2006). Gender differentials in cognitive impairment and decline of the oldest old in China. J. Gerontol. B. Psychol. Sci. Soc. Sci. 61, S107-S115. doi: 10.1093/geronb/61.2.s107

\section{Conflict of Interest: XJ and XZ were employed by MindRank AI Ltd.}

The remaining authors declare that the research was conducted in the absence of any commercial or financial relationships that could be construed as a potential conflict of interest.

Publisher's Note: All claims expressed in this article are solely those of the authors and do not necessarily represent those of their affiliated organizations, or those of the publisher, the editors and the reviewers. Any product that may be evaluated in this article, or claim that may be made by its manufacturer, is not guaranteed or endorsed by the publisher.

Copyright (C) 2022 Jin, Long, Chen, Zeng, Zhang, Yan and Wu. This is an openaccess article distributed under the terms of the Creative Commons Attribution License (CC BY). The use, distribution or reproduction in other forums is permitted, provided the original author(s) and the copyright owner(s) are credited and that the original publication in this journal is cited, in accordance with accepted academic practice. No use, distribution or reproduction is permitted which does not comply with these terms. 